\title{
Pacific
}

Journal of

Mathematics

\section{HECKE CHARACTERS OF SINGULAR DRINFEL'D MODULES}

SUNGHAN BAE 


\title{
HECKE CHARACTERS ()F SINGULAR DRINFELD MODULES
}

\author{
SUNGHAN BAE
}

\begin{abstract}
The $j$-invariant $j$ of a Drinfeld module of rank 2 on $\mathbf{F}_{q}[T]$ over $C$ determine an isomorphism class of Drinfeld modules over $C$. But for singular Drinfeld modules the pair $(j, \chi)$ of a singular $j$-invariant $j$ and an algebraic Hecke character $\chi$ represent an $H$-isomorphism class of singular Drinfeld modules, where $H$ is the Hilbert class field of certain imaginary quadratic function field.
\end{abstract}

0. Introduction. In the theory of elliptic curves (or more generally, abelian varieties) with complex multiplication, the Hecke characters play some important roles, such as the classification of isogeny classes of elliptic curves and the study of zeta functions. In the theory of Drinfeld modules, Gross introduced the notion of algebraic Hecke characters $[\mathbf{G r} \mathbf{1}]$. In this note we restrict ourselves to the Hecke characters arising from the singular Drinfeld modules of rank 2 on $A=\mathbf{F}_{q}[T]$, and see the correspondences between isogeny classes or isomorphism classes of singular Drinfeld modules and Hecke characters.

We fix the following notations throughout this paper:

$\mathbf{F}_{q}$ : finite field of $q$-elements

$A=\mathbf{F}_{q}[T], k=\mathbf{F}_{q}(T)$

$K=$ quadratic extension of $k$ where $\infty$ does not split

$L^{s}=$ separable closure of a field $L$

$\bar{L}=$ algebraic closure of a field $L$.

1. Hecke Characters and Frobenius morphism. Let $L$ be an $A$-field. In this note by a Drinfeld module over $L$ we always mean a Drinfeld module of rank 2 on $A$. Thus a Drinfeld module $\phi$ is completely determined by

$$
\phi_{T}(X)=T X+g X^{q}+\Delta X^{q^{2}}
$$


with $g, \Delta \in L$.

From now on, unless otherwise stated, we suppose that $L$ is a global function field, that is, a finite extension of $k$ and that $\phi$ is a singular Drinfeld module with $\operatorname{End}(\phi)$ isomorphic to an order $\mathcal{O}$ of an imaginary quadratic function field $K$. We fix an isomorphism

$$
\theta: K \longrightarrow \operatorname{End}(\phi)_{k}=\operatorname{End}(\phi) \otimes_{A} k
$$

so that $\frac{d \theta(\alpha)}{d X}=\alpha$ for $\alpha \in \mathcal{O}$, viewing $\theta(\alpha)$ as an additive polynomial in $X$. Such an isomorphism $\theta$ is said to be normalized. (See $[\mathbf{B}-\mathbf{K}]$ for more details.)

LEMMA 1.1. $K$ is contained in $L$ if and only if every element in $\operatorname{End}(\phi)$ is defined over $L$.

Proof. Let $\sigma \in \operatorname{Aut}\left(L^{s} / L\right)$ and $u \in \mathcal{O}$. Then we have

$$
\frac{d \theta(u)^{\sigma}}{d X}=u^{\sigma}
$$

Hence it follows from [B-K], Prop. 2.2, that $\theta(u)^{\sigma}=\theta(u)$ if and only if $u^{\sigma}=u$. Thus the result follows.

By the analytic description of Drinfeld modules via lattices, there is an $A$-lattice $\mathfrak{a}$ of $K$ and an $A$-module isomorphism

$$
\alpha: K / \mathfrak{a} \stackrel{\sim}{\longrightarrow} \operatorname{Tor}(\phi) .
$$

Then one can follow the methods in $[\mathbf{L}]$, Chap. 10, $\S 4$, to get

Theorem 1.2. Assume $K \subset L$. Let $I_{L}$ be the set of ideles of $L$. Then,

(i) $L($ Tor $\phi)$ is abelian over $L$ and there is a unique homomorphism

$$
\chi_{\phi}: I_{L} \longrightarrow K^{*}
$$




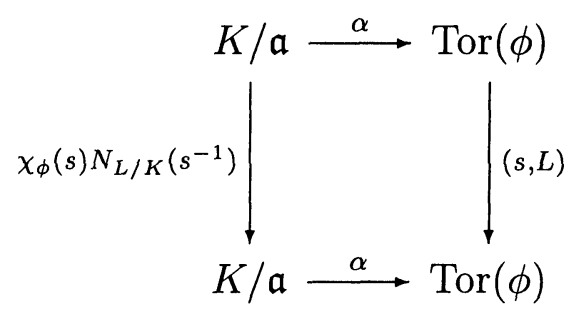

where $(s, L)$ is the Artin map.

(ii) $\chi_{\phi}$ has finite order on the subgroup $\Pi_{v \mid \infty} L_{v}^{*} \times \Pi_{v \nmid \infty} \mathcal{O}_{L, v}^{*}$, and $\chi_{\phi}(s)=N_{L / K}(s)$ for $s \in L^{*}$.

(iii) If $\mathcal{P}$ is a prime ideal of $\mathcal{O}_{L}$ where $\phi$ has good reduction, then $\chi_{\phi}$ is unramified at $\mathcal{P}$, that is, $\chi_{\phi}$ is trivial on local $\mathcal{P}$-units. In this case $\chi_{\phi}(\mathcal{P})$ is well-defined and the reduction $\overline{\theta\left(\chi_{\phi}(\mathcal{P})\right)}$ of $\theta\left(\chi_{\phi}(\mathcal{P})\right)$ at $\mathcal{P}$ is the Frobenius homomorphism of the reduced Drinfeld module $\bar{\phi}$ at $\mathcal{P}$.

Proof. Let $\sigma$ be an automorphism of $L(\operatorname{Tor}(\phi))$ over $L$ inducing $(s, L)$ on the maximal abelian subfield of $L(\operatorname{Tor}(\phi))$. Then the restriction of $\sigma$ to $K^{a b}$ is equal to

$$
\left(N_{L / K}(s), K\right) \text {. }
$$

Let $t=N_{K / L}(s)$. Then applying (1.12) of [Ge], we get an $A$-module isomorphism

$$
\beta: K / t^{-1} \mathfrak{a} \stackrel{\sim}{\longrightarrow} \operatorname{Tor}\left(\phi^{\sigma}\right)
$$

making the following diagram commutative with $\phi^{\sigma}=\sigma$, since $\phi$ is defined over $L$;

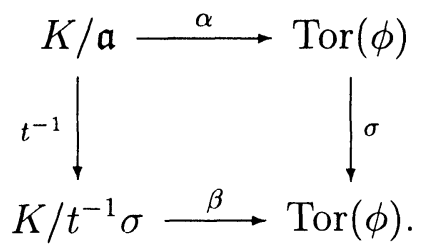

Now the rest of the proof is just the translation of the proofs of Theorems 8 and 9 of $[\mathbf{L}]$, Chap. 10, $\S 4$ into the Drinfeld setting. Note that $\chi_{\phi}(s)$ in this note is $\mu(s)$ in the notation of $[\mathbf{L}]$. 
REMARKS. 1. Above theorem implies that $\chi$ is an algebraic Hecke character of weight 1 in the sense of Gross [Gr1].

2. It is easy to see that $\chi_{\phi}=\chi_{\phi^{\prime}}$ if and only if $\phi$ and $\phi^{\prime}$ are isogenous over $L$.

Nowwe will consider the case $K \not \subset L$. For this we need some properties of reductions of Drinfeld modules.

Proposition 1.3. Let $M$ be a complete field with discrete valuation $v, R$ its ring of integers and $\mathfrak{m}$ its maximal ideal. Suppose that the residue field $R / \mathfrak{m}$ is with characteristic $\mathcal{P} \subset A$, and $a \in A \backslash \mathcal{P}$. Let $\tilde{v}$ be the extension of $v$ on $M^{s}$. Put

$$
\begin{aligned}
& R^{s}=\left\{x \in M^{s}: \tilde{v}(x) \geq 0\right\} \\
& \mathfrak{m}^{s}=\left\{x \in R^{s}: \tilde{v}(x)>0\right\} .
\end{aligned}
$$

Let $\phi$ be a Drinfeld module of rank 2 defined over $R$. Then

(i) $\operatorname{Tor}_{\phi}\left(\mathfrak{m}^{s}\right)=\left\{x \in \mathfrak{m}^{s}: x \in \operatorname{Tor}(\phi)\right\}$ has no nontrivial points of order $a$.

(ii) If $\phi$ has good reduction $\bmod \mathfrak{m}$, then

$$
\operatorname{Tor}_{\phi}(R)_{a}=\left\{x \in R^{s}: \phi_{a}(x)=0\right\} \longrightarrow \operatorname{Tor}_{\phi} \overline{(R / \mathfrak{m})}
$$

is injective and $M\left(\operatorname{Tor}_{\phi}\left(R^{s}\right)_{a}\right)$ is an unramified extension of $M$.

Proof. (ii) follows easily from (i). To show (i) let $x$ be a nonzero element in $\mathrm{m}^{s}$. Then

$$
\tilde{v}\left(\phi_{a}(x)\right)=\tilde{v}(a x)=\tilde{v}(x)>0, \text { since } \phi_{a}(x) \equiv a x \quad\left(\bmod x^{q}\right) .
$$

Hence $\phi_{a}(x) \neq 0$.

Lemma 1.4. Let the notations be the same as in Proposition 1.3. Assume that $\phi$ has good reduction. Then the reduction map

$$
\operatorname{End}(\phi) \longrightarrow \operatorname{End}(\bar{\phi})
$$

is well-defined and injective.

Proof. This follows from (3.3) of $[\mathrm{Ge}]$.

We now go back to our situation. 
THEOREM 1.5. Let $\phi$ be defined over a global function field $L_{0}$ not containing $K$. Let $L=L_{0} K$. Let $\mathcal{P}_{0}$ be a prime ideal of $\mathcal{O}_{L_{0}}$ where $\phi$ has good reduction. Let

$$
\sigma: \xi \longmapsto \xi^{\prime}
$$

be the automorphism of $L$ over $L_{0}$ and $\mathcal{P}$ and $\mathcal{P}^{\prime}$ be the primes of $L$ above $\mathcal{P}_{0}$. Let $\chi_{\phi}(\mathcal{P})$ be defined as in Theorem 1.2 taking $\phi$ to be defined over $L$. Then

(i) $\mathcal{P}_{0}$ is unramified in $L$.

(ii) $\chi_{\phi}(\mathcal{P})^{\prime}=\chi_{\phi}\left(\mathcal{P}^{\prime}\right)$. Let $\Pi_{\mathcal{P}_{0}}$ (resp. $\Pi_{\mathcal{P}}$ and $\left.\Pi_{\mathcal{P}^{\prime}}\right)$ be the Frobenius endomorphism of the reduction $\bar{\phi}\left(\mathcal{P}_{0}\right)$ at $\mathcal{P}_{0}($ resp. $\bar{\phi}(\mathcal{P})$ and $\bar{\phi}\left(\mathcal{P}^{\prime}\right)$ at $\mathcal{P}$ and $\mathcal{P}^{\prime}$ viewing $\phi$ over $\left.L\right)$. Let $(p(T))=N_{L_{0} / k}\left(\mathcal{P}_{0}\right)$ where $p(T)$ is a monic polynomial in $A$.

(iii) If $\mathcal{P}_{0}$ remain prime in $L$, then $\Pi_{\mathcal{P}_{0}}$ is not rational and we have

$$
\Pi_{\mathcal{P}}=\Pi_{\mathcal{P}_{0}}^{2} \text { and } \Pi_{\mathcal{P}}^{2}=\bar{\phi}_{\mu p(T)} \text { with } \mu \in \mathbf{F}_{q}^{*} \text {. }
$$

In this case $\bar{\phi}\left(\mathcal{P}_{0}\right)$ is supersingular.

(iv) If $\mathcal{P}_{0}$ splits completely in $L$, then

$$
\begin{aligned}
\Pi_{\mathcal{P}_{0}} & =\Pi_{\mathcal{P}} \\
\Pi_{\mathcal{P}_{0}} & =\overline{\theta\left(\chi_{\phi}(\mathcal{P})\right)} \text { and } \Pi_{\mathcal{P}_{0}}^{\prime}=\overline{\theta\left(\chi_{\phi}\left(\mathcal{P}^{\prime}\right)\right)}
\end{aligned}
$$

In this case $\bar{\phi}\left(\mathcal{P}_{0}\right)$ is not supersingular.

Proof. (i) follows from Lemma 1.4. Exactly the same argument as in $[\mathbf{L}]$ would prove (ii). If $\mathcal{P}_{0}$ remain prime, then $\mathcal{P}=\mathcal{P}^{\prime}$ and $\Pi_{\mathcal{P}_{0}}^{2}=\Pi_{\mathcal{P}}$. Thus $\chi_{\phi}(\mathcal{P})$ is fixed by $\sigma$ and hence $\chi_{\phi}(\mathcal{P})$ is rational. Hence $\Pi_{\mathcal{P}}=\bar{\phi}_{\mu p(T)}$ where $\mu \in \mathbf{F}_{q}^{*}$, and this proves (iii).

Now suppose that $\mathcal{P}_{0}$ splits completely in $L$. Then $\Pi_{\mathcal{P}_{0}}=\Pi_{\mathcal{P}}$. Thus $\Pi_{\mathcal{P}_{0}}$ is the reduction of $\theta\left(\chi_{\phi}(\mathcal{P})\right) \bmod \mathcal{P}$. Furthermore

$$
\theta\left(\chi_{\phi}(\mathcal{P})\right) \theta\left(\chi_{\phi}\left(\mathcal{P}^{\prime}\right)\right)^{\prime}=\phi_{N_{K / k}\left(\chi_{\phi}(\mathcal{P})\right)} .
$$

Taking bar, we get

$$
\Pi_{\mathcal{P}_{0}}^{\prime}=\overline{\theta\left(\chi_{\phi}(\mathcal{P})^{\prime}\right)}
$$


2. Twists of Drinfeld modules. Let $j(\phi)$ denote the $j$-invariant of a Drinfeld module $\phi$.

THeOREM 2.1. Let $\phi$ be a Drinfeld over a field L. Then there is a bujection between the sets

$$
\left\{\begin{array}{l}
\text { Isomorphism classes } \psi \\
\text { over } L \text { with } j(\psi)=j(\phi)
\end{array}\right\} \longleftrightarrow H^{1}\left(G, \text { Aut }_{I^{s}}(\phi)\right)
$$

which takes $\phi$ to the trivial class. Here $G=\operatorname{Gal}\left(L^{s} / L\right)$.

Proof. Suppose that $j(\phi) \neq 0$. Then $\operatorname{Aut}_{L^{s}}(\phi)=\mathbf{F}_{q}^{*}$. If $j(\psi)=$ $j(\phi)$, we can choose an element $c \in \bar{L}$ such that

$$
c \phi c^{-1}=\psi
$$

Then $c^{q-1} \in L$, since $j(\phi) \neq 0$. Hence $c \in L^{s}$. Define a map)

$$
\eta_{\psi}: G \longrightarrow \operatorname{Aut}_{L^{s}}(\phi)=\mathbf{F}_{q}^{*}
$$

by $\eta_{\psi}(\sigma)=\sigma(c) / c$. Since $c^{q-1} \in L, \sigma(c)=\mu c$ with $\mu \in \mathbf{F}_{q}^{*}$ by Kummer theory. Hence $\sigma(c) / c \in \mathbf{F}_{q}^{*}$ and $\eta_{\psi}$ is well-defined and gives an element of $H^{1}\left(G, \operatorname{Aut}_{L^{s}}(\phi)\right)$.

Conversely, let

$$
\eta: G \longrightarrow \operatorname{Aut}_{L^{s}}(\phi)=\mathbf{F}_{q}^{*}
$$

be a cocycle. Since $H^{1}\left(G,\left(L^{s}\right)^{*}\right)$ is trivial and $\mathbf{F}_{q}^{*} \subset\left(L^{s}\right)^{*}$, there is an element $c \in\left(L^{s}\right)^{*}$ such that

$$
\eta(\sigma)=\sigma(c) / c .
$$

Since $\sigma(c) / c \in \mathbf{F}_{q}^{*}, \sigma\left(c^{q-1}\right)=c^{q-1}$. Thus $c^{q-1} \in L$ and the Drinfeld module $\phi^{\eta}=c \phi c^{-1}$ is defined over $L$. It is easy to see that the maps

$$
\psi \longmapsto \eta_{\psi} \text { and } \eta \longmapsto \phi^{\eta}
$$

are inverses to each other.

If $j(\phi)=0$, then $\operatorname{Aut}_{L^{s}}(\phi)=\mathbf{F}_{q^{2}}^{*}$. But the same proof as above will give the results in this case too.

We call $\phi^{\eta}$ the twist of $\phi$ assosiated to $\eta \in H^{1}\left(G, \operatorname{Aut}_{L^{s}}(\phi)\right)$. 
REMARK. Theorem 2.1 holds for Drinfeld modules which are not singular.

Now we will consider the action of the twists on the Hecke characters. Let $L=H$ be the Hilbert class field of $K$, that is, the maximal unramified abelian extension of $K$ where $\propto$ splits completely. It is well-known that $H=K(j(\phi))$ for any Drinfeld module $\phi$ with complex multiplication by $K$. Let $G=\operatorname{Gal}\left(H^{s} / H\right)$. As in the proof of Theorem 2.1, each $\eta \in H^{1}\left(G, \operatorname{Aut}_{I^{s}}(\phi)\right)$ corresponds to an element $c(\eta)$ of $\left(H^{s}\right)^{*}$ such that

$$
\phi^{\prime \prime}=r \cdot(\eta) \phi c(\eta)^{-1}
$$

We also know that

$$
c(\eta)^{q-1} \in H \text { if } j(\phi) \neq 0
$$

and

$$
c(\eta)^{q^{2}-1} \in H \text { if } j(\phi)=0 .
$$

Thus we can identify $H^{1}\left(G, \operatorname{Aut}_{H^{s}}(\phi)\right)$ with

$$
\begin{aligned}
& \left(H^{\frac{1}{q-1}}\right)^{*} / H^{*} \text { if } j \neq 0 \\
& \left(H^{\frac{1}{q^{2}-1}}\right)^{*} / H^{*} \text { if } j=0 .
\end{aligned}
$$

From now on, we assume that $\operatorname{End}_{C}(\phi)$ is isomorphic to $\mathcal{O}_{K}$, the ring of integers of $K$. Since $K \subset H$, any endomorphism of $\phi$ is defined over $H$ by Lemma 1.1. Hence any class $\eta$ in $H^{1}\left(G, \operatorname{Aut}_{I^{s}}(\phi)\right)$ is represented by a continuous homomorphism

$$
\eta: G \longrightarrow \mathcal{O}_{K}^{*}
$$

Here $\eta(\sigma)=\sigma(c(\eta)) / c(\eta)$ and

$$
\mathcal{O}_{K}^{*}=\left\{\begin{array}{l}
\mathbf{F}_{q}^{*}, \text { if } j(\phi) \neq 0 \\
\mathbf{F}_{q^{2}}^{*}, \text { if } j(\phi)=0 .
\end{array}\right.
$$

The Artin homomorphism

$$
(, H): I_{H} / H^{*} \longrightarrow G^{a b}
$$


allows us to view $\eta$ as a continuous homomorphism

$$
\underline{\eta}=\eta \circ(, H): I_{H} \longrightarrow K^{*}
$$

which is trivial on the principal ideles.

Proposition 2.2. For $\eta \in H^{1}\left(G, \operatorname{Aut}_{H^{s}}(\phi)\right), \phi^{\eta}$ has complex multiplication by $\mathcal{O}_{K}$ over $H$ and

$$
\chi_{\phi^{\eta}}=\underline{\eta} \cdot \chi_{\phi}
$$

Proof. Since $\phi^{\eta}=c(\eta) \cdot \phi \cdot c(\eta)^{-1}$,

$$
\operatorname{End}_{\bar{H}}\left(\phi^{\eta}\right)=c(\eta) \cdot \operatorname{End}_{\bar{H}}(\phi) \cdot c(\eta)^{-1} .
$$

Let $u \in \operatorname{End}_{\bar{H}}(\phi)$. Then $u=\sum a_{\imath} \tau^{i}$ with $a_{i} \in H$ where $\tau(x)=x^{q}$. Then

$$
c(\eta) \cdot u \cdot c(\eta)^{-1}=\sum a_{i} c(\eta)^{1-q^{i}} \tau^{i} .
$$

Hence $c(\eta) \cdot u \cdot c(\eta)^{-1}$ is defined over $H$ by the construction of $c(\eta)$ if $j(\phi) \neq 0$. If $j(\phi)=0$, it is easy to see that $a_{i}=0$ for odd $i$. Hence $c(\eta) \cdot u \cdot c(\eta)^{-1}$ is defined over $H$ in this case too.

Consider the following commutative diagram in Theorem 1.2,

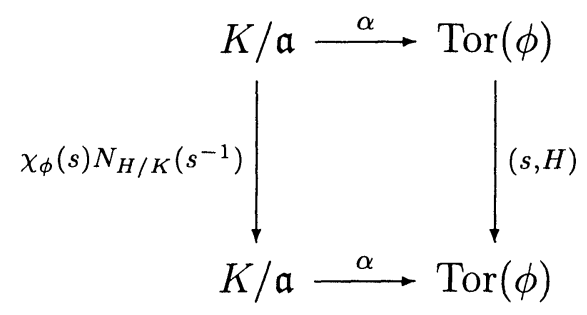

and

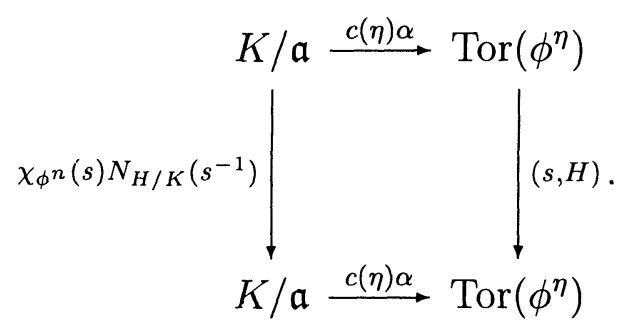


Assume that $j(\phi) \neq 0$.

We have, for $x \in K / \mathfrak{a}$,

$$
\begin{aligned}
(c(\eta) \alpha(x))^{(s, H)} & =c(\eta)^{(s, H)} \alpha(x)^{(s, H)} \\
& =c(\eta)^{(s, H)} \alpha\left(\chi_{\phi}(s) N_{H / K}\left(s^{-1}\right) x\right) \\
& =c(\eta) \frac{c(\eta)^{(s, H)}}{c(\eta)} \alpha\left(\chi_{\phi}(s) N_{H / K}\left(s^{-1}\right) x\right) .
\end{aligned}
$$

If $j(\phi) \neq 0$, then the last equation is equal to

$$
c(\eta) \cdot \alpha\left(\eta((s, H)) \cdot \chi_{\phi}(s) N_{H / K}\left(s^{-1}\right) x\right)
$$

since $\eta((s, H))=\frac{c(\eta)^{(s, H)}}{c(\eta)} \in \mathbf{F}_{q}^{*}$. Therefore, from the latter diagram,

$$
\chi_{\phi^{\eta}}(s)=\underline{\eta}(s) \chi_{\phi}(s) .
$$

If $j(\phi)=0$, then $K=\mathbf{F}_{q^{2}}(T)$ and $\alpha$ is $\mathbf{F}_{q^{2}}$-linear. Hence the result follows in this case too.

Lemma 2.3. Let $\phi$ and $\psi$ be two singular Drinfeld modules over $H$ with complex multiplication by $\mathcal{O}_{K}$. Then they become isogenous over $H^{s}$.

Proof. It is easy to see that $\phi$ and $\psi$ are isogenous over $\bar{H}$. Let

$$
u: \phi \longrightarrow \psi
$$

be an isogeny over $\bar{H}$. Write

$$
\begin{gathered}
u=\sum_{i=0}^{r} a_{i} \tau^{\imath}, \\
\phi_{T}=T+g \tau+\Delta \tau^{2}
\end{gathered}
$$

and

$$
\psi_{T}=T+g^{\prime} \tau+\Delta^{\prime} \tau^{2}
$$

where $g, g^{\prime}, \Delta$ and $\Delta^{\prime}$ are in $H$.

Then

$$
a_{r} \Delta^{q^{r}}=a_{r}^{q^{2}} \Delta^{\prime}
$$


Hence $a \in H^{s}$. One can use induction to show that $a_{r-i} \in H^{s}$ for every $i \geq 0$. Hence $\phi$ and $\psi$ are isogenous over $H^{s}$.

Suppose that $\phi$ and $\psi$ are two singular Drinfeld modules over $H$ with complex multiplication by $\mathcal{O}_{K}$. Choose an isogeny

$$
u: \phi \longrightarrow \psi
$$

over $H^{s}$. Let $A$-deg $u$ denote the monic generator of the $A$-degree ideal of $u$ (see $[\mathbf{B}-\mathbf{K}]$ for the definition of the $A$-degree ideal), and $\breve{u}$ denote the isogeny

$$
\breve{u}: \psi \longrightarrow \phi
$$

such that

$$
\breve{u} \circ u=\phi_{A-\operatorname{deg} u}
$$

and

$$
u \circ \breve{u}=\psi_{A-\operatorname{deg} u} .
$$

Clearly $\breve{u}$ is defined over $H^{s}$.

Define a 1-cocycle

$$
\begin{aligned}
\eta: G=\operatorname{Gal}\left(H^{s} / H\right) & \longrightarrow\left(\operatorname{End}_{I^{s}}(\phi) \otimes_{A} k\right)^{*} \\
\sigma & \longmapsto \breve{u} \circ \sigma(u) \otimes \frac{1}{A-\operatorname{deg} u}=\breve{u} \circ \sigma(u) \circ \phi_{A-\operatorname{deg} u}^{-1}
\end{aligned}
$$

It is easy to see that $\eta$ is continuous and independent of the choice of $u$. As before one can define the induced homomorphism

$$
\underline{\eta}: I_{H} \longrightarrow \mathcal{O}_{K}^{*} \text {. }
$$

Then the same proof as in Proposition 2.2 gives,

Proposition 2.4. Let $\phi$. a and $\eta$ are sa above. Then,

$$
\backslash_{\imath}=\underline{\eta} \cdot \backslash_{\phi}: I_{I I} \longrightarrow K^{*}
$$


3. Drinfeld Modules over $H$. To each singular Drinfeld module $\phi$ over $H$ with complex multiplication by $\mathcal{O}_{K}$, we have associated two invariants :

$$
\begin{aligned}
& \text { the } j \text {-invariant } j(\phi) \in H \\
& \text { the Hecke character } \chi_{\phi}: I_{H} \longrightarrow K^{*} \text {. }
\end{aligned}
$$

The $j$-invariants of such Drinfeld modules form a finite set $\mathcal{J}$ of cardinality $h=\# \mathrm{Cl}\left(\mathcal{O}_{K}\right)$. We have the following classification theorem for singular Drinfeld modules over $H$, whose proof is exactly the same as in the classical case [Gr2].

\section{THEOREM 3.1.}

(a) Let $j$ be an element of $\mathcal{J}$ and let

$$
\chi: I_{H} \longrightarrow K^{*}
$$

be a homomorphism whose restriction to $H^{*}$ is the norm, and satisfies the condition that $\chi$ has finite order on the subgroup

$$
\Pi_{v \mid \infty} H_{v}^{*} \times \Pi_{v \nmid \infty} \mathcal{O}_{H, v}^{*}
$$

Then there is a singular Drinfeld module $\phi$ with complex multiplication by $\mathcal{O}_{K}$ over $K$ with $j(\phi)=j$ and $\chi_{\phi}=\chi$.

(b) The character $\chi_{\phi}$ determines the isogeny class of $\phi$ over $H$, and the pair $\left(j(\phi), \chi_{\phi}\right)$ determines the isomorphism class of $\phi$ over $H$. Two singular Drinfeld modules are isomorphic over $H$ if they are isogeneous over $H$ and isomorphic over $H^{s}$.

Proof. Given $j$ and $\chi$, let $\psi$ be any Drinfeld module over $H$ with $j(\psi)=j$. Let

$$
\underline{\eta}=\chi / \chi_{\psi}: I_{H} \longrightarrow K^{*}
$$

Then $\underline{\eta}$ is trivial on $H^{*}$, and thus induces

$$
\eta: G=\operatorname{Gal}\left(H^{s} / H\right) \longrightarrow K^{*}
$$

via the Artin isomorphism. Since $\underline{\eta}$ is of finite order on $\Pi_{v \mid \infty} H_{v}^{*} \times$ $\Pi_{v \nmid \infty} \mathcal{O}_{H, v}^{*}$, the image of $\eta$ lies in $\mathcal{O}_{K}^{*}=\mu\left(K^{*}\right)$. Then by Proposition 2.2 , the Drinfeld module $\phi=\psi^{\eta}$ has complex multiplication by $\mathcal{O}_{K}$ with $j(\phi)=j(\psi)=j$ and $\chi_{\phi}=\chi_{\psi} \cdot \underline{\eta}=\chi$. But Proposition 2.4 
implies that $\chi_{\phi}$ determines $\phi$ up to isogeny over $H$. By Proposition 2.2 , the pair $\left(j, \chi_{\phi}\right)$ determines $\phi$ up to $H$-isomorphisms.

REMARK. The condition that $\chi$ has finite order on the subgroup

$$
\Pi_{v \mid \infty} H_{v}^{*} \times \Pi_{v \nmid \infty} \mathcal{O}_{I I, v}^{*}
$$

is stronger than the condition that $\chi$ is continuous. The reason for this condition is that $I_{I} / H^{*}$ contains a subgroup isomorphic to $\mathbf{Z}$.

Next we will consider the action of the group $\operatorname{Gal}(H / k)$. Let $\sigma \in \operatorname{Gal}(H / K)$. The action of $\sigma$ on $\chi_{\phi}$ is given by

$$
\sigma\left(\chi_{\phi}\right)(s)=\sigma\left(\chi_{\phi}\left(\sigma^{-1} s\right)\right)
$$

Exactly the same proof as in the classical theory would give

$$
\text { LEMMA 3.2. If } \psi=\phi^{\sigma} \text {, then } \chi_{\psi}=\sigma\left(\chi_{\phi}\right) \text {. }
$$

Let $\phi$ and $\psi$ be two singular Drinfeld modules with complex multiplication by $\mathcal{O}_{K}$. The group $\operatorname{Hom}_{H}(\phi, \psi)$ is trivial unless $\chi_{\phi}=\chi_{\psi}$ by Proposition 2.2. Hence assume that $\chi_{\phi}=\chi_{\psi}$. Then, by Proposition 2.4,

$$
\operatorname{Hom}_{H}(\phi, \psi)=\operatorname{Hom}_{I^{s}}(\phi, \psi) .
$$

Let the association $[\mathfrak{a}] \mapsto \sigma_{[\mathfrak{a}]}$ be the Artin isomorphism

$$
\mathrm{Cl}\left(\mathcal{O}_{K}\right) \stackrel{\sim}{\longrightarrow} \operatorname{Gal}(H / K) .
$$

Then

$$
j(\psi)=\sigma_{[\mathfrak{a}]}(j(\phi))
$$

for some integral ideal $\mathfrak{a}$ of $\mathcal{O}_{K}$. Then we have

LEMMA 3.3. If $\chi_{\phi}=\chi_{\psi}$ and $j(\psi)=\sigma_{[\mathfrak{a}]}(j(\phi))$, then the group $\operatorname{Hom}_{I I}(\psi, \phi)$ becomes a projective $\mathcal{O}_{K}$-module of rank 1 . One has an isomorphism

$$
\begin{aligned}
& \mathfrak{a} \stackrel{\sim}{\longrightarrow} \operatorname{Hom}_{H}(\psi, \phi) \\
& \alpha \longmapsto u_{\alpha}
\end{aligned}
$$


where the A-degree ideal of $u_{\alpha}$ is equal to

$$
\left(N_{K / k} \cdot()\left(N_{K / k} \mathfrak{a}\right)^{-1}\right. \text {. }
$$

Here the $\mathcal{O}_{k}-$ module structure as ginen by the isomorphism

$$
\text { (): } \mathcal{O}_{K} \simeq \operatorname{End}_{H}(o)
$$

4. Descended Drinfeld modules. Let o be a singular Drinfeld module with complex multipliation by $\mathcal{O}_{h}$ and let $H_{0}=h \cdot(j(\phi))$. Then by $[\mathrm{Ge}](1.5)$ that $\left[H: H_{0}\right]=2$. Let

$$
\operatorname{Cial}\left(H / H_{0}\right)=\langle\sigma\rangle \text {. }
$$

Wi say that o (an be descended to $H_{0}$ if there is a Drinfeld module b, over $H_{0}$ which is isomorphic to o orer $H$. Then we have.

Proposition 4.1. The singular Drinfeld module os can be descended to $H_{0}$. if and only if $\sigma(j(\phi))=j(\phi)$ and $\sigma\left(\gamma_{\phi}\right)=\chi_{\phi}$.

Proof. The 'only if' part is easy and the same as in the classical (*asce.

Now assume that $j(\phi)$ and $\chi_{\phi}$ are invariant under $\sigma$. Suppose that $j(\phi) \neq 0$. Let $G=\operatorname{Gal}\left(H_{0}^{s} / H_{0}\right)$ and $G_{0}=\operatorname{Gal}\left(H_{0}^{s} / H\right)$. (Once we have identified $H^{1}\left(G, \mathcal{O}_{K^{*}}^{*}\right)$ (resp). $H^{1}\left(G_{0}, \mathcal{O}_{K^{\prime}}^{*}\right)$ ) with $\left(H_{0}^{\frac{1}{q-1}}\right)^{*} / H_{0}^{*}$ (resp. $\left.\left(H^{\frac{1}{q-1}}\right)^{*} / H^{*}\right)$. Then the map

$$
\left(H_{0}^{\frac{1}{q-1}}\right)^{*} / H_{0}^{*} \longrightarrow\left(H^{\frac{1}{4-1}}\right)^{*} / H^{*}
$$

associated to the restriction map

$$
H^{1}\left(G, \mathcal{O}_{K}^{*}\right) \longrightarrow H^{1}\left(G_{0}, \mathcal{O}_{K}^{*}\right)
$$

is just the map induced by the inclusion

$$
\left(H_{0}^{\frac{1}{q-1}}\right)^{*} \longrightarrow\left(H^{\frac{1}{q-1}}\right)^{*}
$$

Since $j(\phi)$ lies in $H_{0}$, we can find a Drinfeld module $\psi$ over $H_{0}$ with $j(\psi)=j(\phi)$. Then there is an element $c \in\left(H^{\frac{1}{q-1}}\right)^{*}$ such that

$$
\phi=c \psi c^{-1}
$$


Let $[r]$ denote the elass of 6 in $\left(H^{\frac{1}{4-1}}\right)^{*} / H^{*}$. Then the action of $\sigma$ on $[$ c] is as follow's :

Lect $d=a^{q-1} \in H$ and $\sigma(c)$ be any $(q-1)$ st root of $\sigma(d)$. Then define

$$
\sigma([r])=[\sigma(r)]
$$

Suppose that $\sigma\left(\backslash_{0}\right)=\lambda_{0}$. Then $\backslash_{0} / \lambda_{0}$. lies in the subgroup $H^{1}\left(G_{0} \cdot \mathcal{O}_{K^{*}}^{*}\langle\sigma\rangle\right.$ and $\backslash_{\varphi} / \lambda_{1}$ (corresponds to $[c]$. Hence $[c]==[\sigma(c)]$ in $\left(H^{\frac{1}{4-i}}\right)^{*} / H^{*}$. i.c.. $\cdot=\sigma(r) \cdot h$ with $h \in H$. Thus

$$
d=\sigma(d) h^{q-1}
$$

and so

$$
\sigma(d)=d \sigma(h)^{q-1}
$$

since $\sigma^{2}=i d$ on $H$. Therefore

$$
(h \sigma(h))^{q-1}=1
$$

Thus

$$
h \sigma(h)=\mu \in \mathbf{F}_{q}^{*} .
$$

However $K / k$ is an imaginary quadratic extension, $\mu \in \mathbf{F}_{q}^{2}$, i.e., $\mu=\nu^{2}, \nu \in \mathbf{F}_{q}^{*}$. Then

$$
\frac{h}{\nu} \sigma\left(\frac{h}{\nu}\right)=1 .
$$

Hence by Hilbert Theorem 90,

$$
\frac{h}{\nu}=h_{1} / \sigma\left(h_{1}\right)
$$

for some $h_{1} \in H$. Let

$$
d_{1}=d / h_{1}^{q-1}
$$

Then it is easy to see that

$$
\sigma\left(d_{1}\right)=d_{1} \text {, i.e. } d_{1} \in H_{0} \text {. }
$$

Let $c_{1}$ be any $(q-1)$-th root of $d_{1}$. Then $[c]$ is the image of $\left[c_{1}\right]$ under restriction and $c_{1} \psi c_{1}^{-1}$ is defined over $H_{0}$ and isomorphic to $\phi$ over $H$. 
In the case of $j(\phi)=0, H=K=\mathbf{F}_{q^{2}}(T)$ and $H_{0}=k=\mathbf{F}_{q}(T)$. Thus the proof is easy and left to the reader.

REMARK. The proof of the above theorem is different from that of the classical case because one cannot lift $\sigma$ to $H^{s}$.

Let $G=\operatorname{Gal}\left(H_{0}^{s} / H_{0}\right)$ and $\varepsilon$ be the class in $H^{1}\left(G, \mathcal{O}_{K}^{*}\right)$ which is represented by the quadratic character

$$
\varepsilon: G \longrightarrow \operatorname{Gal}\left(H / H_{0}\right) \hookrightarrow \mathcal{O}_{K}^{*}
$$

Then we have

\section{THEOREM 4.2.}

(a) Two descended Drinfeld modules $\phi$ and $\psi$ are isogenous over $H_{0}$ if and only if $\chi_{\phi}=\chi_{\psi}$.

(b) Within each $H_{0}$-isogeny class $\{\phi\}$ with a fixed $j$-invariant there are exactly $2 H_{0}$ isomorphism classes, represented by the modules $\phi$ and $\phi^{\varepsilon}$. These Drinfeld modules become isomorphic over $H$, where the $H_{0}$-isogeny $u: \phi \rightarrow \phi^{\varepsilon}$ becomes a complex multiplication.

Proof. (a) If $\phi$ and $\psi$ are isogenous over $H_{0}$, then they are isogenous over $H$, so $\chi_{\phi}=\chi_{\psi}$. Now assume $\chi_{\phi}=\chi_{\psi}$. Then $\phi$ and $\psi$, are isogenous over $H$. Let

$$
u: \phi \longrightarrow \psi
$$

be an $H$-isogeny and $\breve{u}$ as in section 2 . Let $a$ be an element of $K^{*}$ such that

$$
\theta(a)=\frac{\breve{u} \circ \sigma(u)}{\phi_{A-\operatorname{deg} u}} \in\left(\operatorname{Endl}_{I I}(\phi) \otimes_{A} k\right)^{*} .
$$

Then

$$
\begin{aligned}
\theta\left(N_{K / k} a\right) & =\theta(a \cdot \sigma(a)) \\
& =\frac{\breve{u} \circ \sigma(u)}{\phi_{A-\operatorname{deg} u}} \cdot \frac{\sigma(\breve{u}) \circ u}{\phi_{A-\operatorname{deg} u}} \\
& =\frac{\breve{u} \circ \sigma(\breve{u} u) \circ u}{\phi_{(A-\operatorname{deg} u)^{2}}} \\
& =\frac{\breve{u} \circ \sigma\left(\psi_{A-\operatorname{deg} u}\right) \circ u}{\phi_{(A-\operatorname{deg} u)^{2}}}
\end{aligned}
$$




$$
\begin{aligned}
& =\frac{\breve{u} \circ \psi_{A-\operatorname{deg} u} \circ u}{\phi_{(A-\operatorname{deg} u)^{2}}}, \text { since } \psi \text { is descended } \\
& =\frac{\breve{u} \circ u \circ \phi_{A-\operatorname{deg} u}}{\phi_{(A-\operatorname{deg} u)^{2}}} \\
& =\frac{\phi_{A-\operatorname{deg} u} \circ \phi_{A-\operatorname{deg} u}}{\phi_{(A-\operatorname{deg} u)^{2}}}=1 .
\end{aligned}
$$

Hence by Hilbert 90, $a=b / \sigma(b)$ for some $b \in K$. Let $u^{\prime}=u \circ \theta(b)$. Then $\sigma\left(u^{\prime}\right)=u^{\prime}$ and so $u^{\prime}$ is defined over $H_{0}$.

(b) Let $G=\operatorname{Gal}\left(H_{0}^{s} / H_{0}\right)$ and $G_{0}=\operatorname{Gal}\left(H_{0}^{s} / H\right)=\operatorname{Gal}\left(H^{s} / H\right)$. Then the same method as in the classical case will give the result.

\section{REFERENCES}

[B-K] S. Bae and J. K. Koo, On the singular Drinfeld modules of rank 2, Math. Z., 210 (1992), 267-276.

[Ge] E-U. Gekeler, Zur arithmetik von Drinfeld moduln, Math. Ann., 262 (1983), 167-182.

[Gr1] B. Gross, Algebraic Hecke Characters for Function Fields, Séminare de Théorie des Nombres, Paris 1980-81, 87-90, Birkhäuser, Boston Basel Stutt gart, 1982.

[Gr2] - Arithmetic of Elliptic Curves with Complex Multiplication, Lecture Note in Math. 776, Springer, Berlin-Heidelberg-New York, 1980.

[L] S. Lang, Elliptue Functions, GTM 112, Springer, New York- BerlinHeidelberg-London-Paris-Tokyo, 1987.

[S-T] J. P. Serre and J. Tate, Good reduction of Abelian varieties, Ann. Math., 88 (1968), 492-J17.

[T] T. Takahashi, Good reduction of elliptic modules, J. Math. Soc. Japan, $34(1982)$. $475-487$.

Received June 16. 1992 and in revised form November 18, 1992. Partially supported by KoSEF Research Grant 91-08-00-07.

Korea Adv. Inst. of Science and Technology

TAE.JON. 3()5-7()1, K()REA 



\section{PACIFIC JOURNAL OF MATHEMATICS}

Volume $167 \quad$ No. $2 \quad$ February 1995

Existence of shortest directed networks in $\mathbb{R}^{2}$

201

MANUEL AlFARO GARCIA

Hecke characters of singular Drinfel'd modules

215

SUNGHAN BAE

Factorization method for a bimeromorphic morphism

231

Jose PEREZ BLANCO

$L^{p}$ estimates for operators associated to flat curves without the Fourier 243 transform

Anthony Carbery, James Thomas Vance, JR., Stephen

WAINGER, DAVID K. WATSON and JAMES WRIGHT

$S$-integer points on elliptic curves

263

ROBERT HOWARD GROSS and JOSEPH SILVERMAN

On metrics defined by modules

JAMES ALLISTER JENKINS

Conditional Wiener integrals. II

ChUll PARK and DAVID LeE SKOUG

On a Plancherel formula for certain discrete, finitely generated, torsion-free nilpotent groups

CAROLYN PFEFFER JOHNSTON

Desingularizations of some unstable orbit closures

MARK STEPHEN REEDER

Determining multiplicities of half-integral weight newforms

Thomas Richard SHEMANSKE and LyNne WALLING

Generation of integral orthogonal groups over dyadic local fields 